\title{
IN-SITU MONITORING OF MICRO-CHEMICAL VAPOR DEPOSITION ( $\mu$-CVD): EXPERIMENTAL RESULTS AND SPICE MODELING
}

\author{
Ronald P. Manginell, James H. Smith, Antonio J. Ricco, \\ Robert C. Hughes, and Daniel J. Moreno \\ Sandia National Laboratories \\ Albuquerque, New Mexico, 87185-1425 \\ Robert J. Huber \\ University of Utah \\ Salt Lake City, Utah, 84112
}

\begin{abstract}
Two new methods of monitoring the thickness of metallic films as deposited on microfabricated bridge structures via microchemical vapor deposition ( $\mu$-CVD) are described. These methods require no additional fabrication to produce four-point resistancebased thickness probes. Instead, the relatively large increase in (a) the heat conductance and (b) the heat capacity of the microbridge/metal system as a result of deposited metal are detected as variations in the heating power provided to the microbridge by the control circuitry.

Experimental results demonstrating these effects are given for a microbridge controlled with a novel constant-resistance circuit. The heat-transfer equations for the microbridge/metal system and the circuit model for the control circuit are then solved using SPICE. This coupled model predicts within $10 \%$ the measured thickness of $\mu-C V D$ platinum.
\end{abstract}

\section{INTRODUCTION}

Recently, $\mu-C V D$ of metals on micromachined devices has been reported $[1,2,3,4]$. In this technique the self heating of microhotplates and microbridges is used to thermally decompose precursors directly on their surface (Figures 1 and 2). The high degree of thermal isolation of these structures confines deposition only to their active regions, and is therefore highly selective. In the absence of this technique, one additional deposition and patteming step would be required in a conventional microfabrication sequence to fabricate such a film [5], and coverage of the underside of suspended structures would be difficult to achieve. Applications of $\mu-C V D$ include noble-metal catalyst deposition for calorimetric gas sensors [3] and metal deposition for conductometric gas sensors $[\mathbf{1 , 2 , 4 ]}$.

In-situ thickness monitoring via measurement of the thinfilm resistance of $\mu-C V D$ metal on microhotplates has been demonstrated $[\mathbf{1 , 2}]$; for this purpose, four-point probes were fabricated. We show that the sensitive heat-transfer properties of microheater platforms such as microhotplates and microbridges can be utilized instead. Given their relatively small values prior to metal deposition, the (a) heat conductance and (b) heat capacity of the microheater/metal system increase significantly with deposited metal quantity and can be detected in situ with appropriate control circuitry. While (a) alters the steady-state heating power requirements of the system, (b) introduces a delay in its transient response to a heating pulse.

These effects are demonstrated experimentally for $\mu-C V D$ of Pt on CMOS-compatible, silicon-nitride-encapsulated, polysilicon microbridges. A constant-resistance control circuit used for microbridge heating during $\mu-C V D$ is presented (Figure 3 ). This circuit provides both steady-state $(\mathrm{CW})$ and pulsed resistance set points so that both heat conductance and capacity changes can be probed. It should be stressed that the techniques described here are applicable to microhotplates as well, and that no additional microfabrication is required, in contrast to four-point resistance probes. Furthermore, in-situ monitoring of the $\mu-C V D$ of dielectrics should also be achievable in a pulsed-bias mode, since this technique only requires changes in heat capacity.

An electro-thermal model of the microbridge/metal system is coupled with the circuit model of the control circuit and solved in SPICE. Boundary conditions, implementations of temperaturevariable resistors, and the conductivity and heat capacity of the deposited metal are described. Simulations predict metal thickness within $10 \%$ of measured values for both $\mathrm{CW}$ and pulsed heating bias.

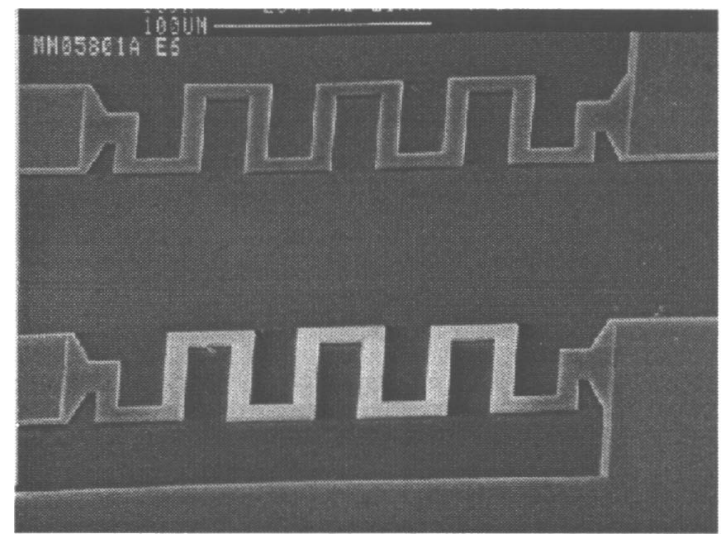

Figure 1. Scanning electron micrograph of two meandered microbridges. The lower bridge appears brighter due to a smooth layer of Pt approximately $0.1 \mu \mathrm{m}$ in thickness, which was deposited by $\mu-C V D$ from the precursor $\mathrm{Pt}(\text { acac })_{2}$.

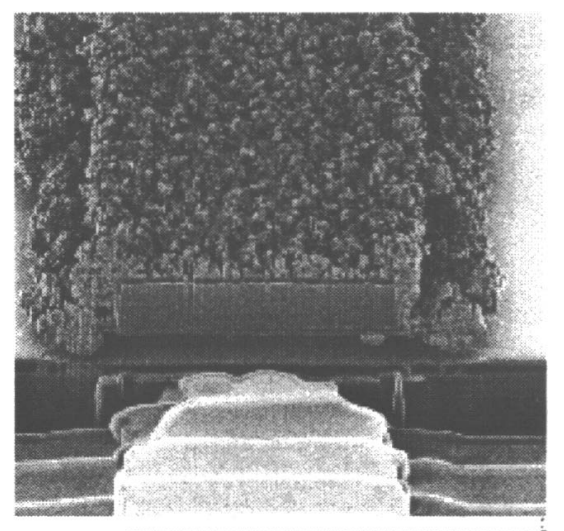

Figure 2. Focused ion beam cross section of a silicon-nitrideencapsulated, polysilicon microbridge with a rough coating of $\mu$ CVD platinum. The microbridge is $2 \mu \mathrm{m}$ thick and $10 \mu \mathrm{m}$ wide. 


\section{THEORY}

As the thickness of metal deposited on a microbridge increases, so does its ability to conduct heat along the device. This means that the power, $P(t)$, required to maintain the bridge/metal system at constant resistance, $R$, increases with deposition time. The relative power provided to the bridge can be written

$$
P(t) / P_{O}=1+P_{m}(t) / P_{O},
$$

where $P_{m}$ is the increasing power conducted by the catalyst and $P_{o}$ is the power required prior deposition. By the chain rule, the rate of increase of $P_{m}$ with metal thickness, $z_{m}$, at constant resistance is

$$
\left.\frac{d P_{m}}{d t}\right|_{R}=\left.\left.\frac{d P_{m}}{d z_{m}}\right|_{R} \cdot \frac{d z_{m}}{d t}\right|_{R} .
$$

The growth rate of the film is then

$$
\left.\frac{d z_{m}}{d t}\right|_{R}=\left.\frac{d P_{m}}{d t}\right|_{R} \cdot\left(\left.\frac{d P}{d z_{m}}\right|_{R}\right)^{-1} .
$$

The composite thermal conductivity $K$ of the microbridge with deposited metal is

$$
K\left(z_{m}\right)=K_{b}+\left[\frac{(2 z+w) z_{m}}{w z}\right] \cdot K_{m} \cdot f,
$$

where $K_{b}, z$ and $w$ are the conductivity, thickness, and width, respectively of the bridge. The factor $f$ accounts for the fact that the deposited film has less than bulk density (Figure 2), so its actual conductance is less than that implied by the bulk conductivity $K_{m}$ of the metal. Finally, the term in brackets is the ratio of cross-sectional area of the deposited film, to that of the bridge, as shown in Figure 4.

The heat capacity of a differential element of the bridge/metal system is the parallel combination of the capacity of the bridge, and the capacity of the metal. For a bridge of length $L$ divided into $N$ elements, the latter term is

$$
C_{m}=f \cdot c_{m} \cdot(2 z+w) \cdot z_{m} \cdot(L / N),
$$

where $c_{m}$ is the bulk specific heat of the metal. The factor $f$ is the same as in Eq. (4).

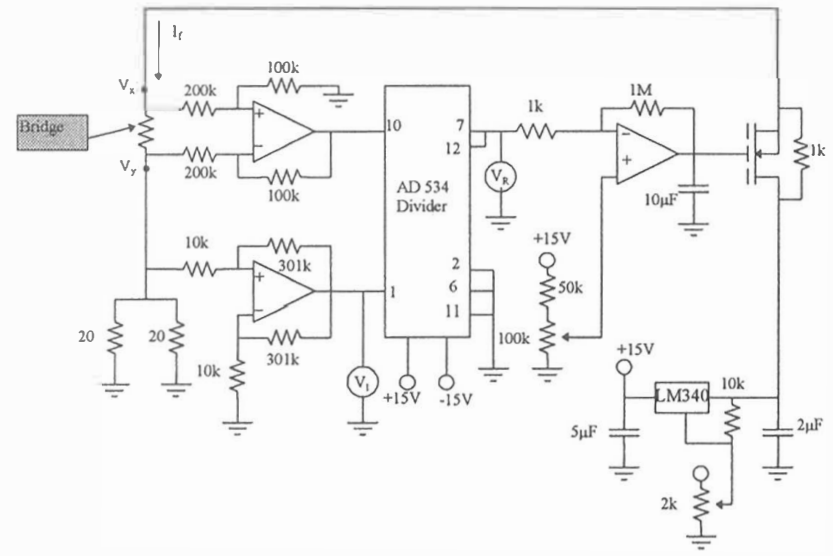

Figure 3. Constant-resistance control circuit. ACW set point is shown, but pulsed operation is also possible.

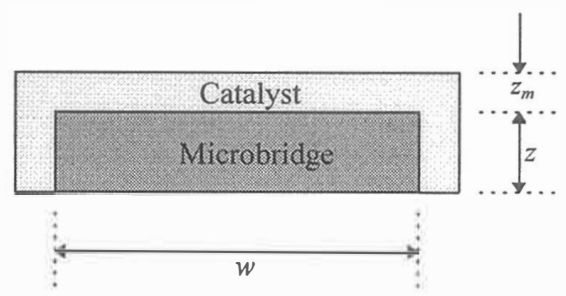

Figure 4. A cross section of the microbridge with deposited metal catalyst. Dimensions of the bridge and assumed average thickness of the catalyst are shown

\section{EXPERIMENTAL DETAILS}

The $\mu-C V D$ apparatus is described elsewhere $[3,6]$. During $\mu-C V D$ of $\mathrm{Pt}$, the microbridge was heated with a constantresistance control circuit (Figure 3 ) and the power provided to the device was monitored. Referring to Figure 3, the resistance and current of the bridge (for the feedback resistor values shown) are

$$
R=60.2 \cdot V_{R} \quad \text { and } \quad I=V_{I} / 0.31 \text {, }
$$

and the power is obtained by the relation $P=I^{2} R$. For $\mathrm{CW}$ biasing, the voltages $V_{R}$ and $V_{l}$ of Eq. (6) were recorded with a digital multimeter; for pulsed biasing, an 8-bit digitizing oscilloscope was used. Note that the polysilicon bridge material is electrically insulated from the deposited metal by a $0.25 \mu \mathrm{m}$ layer of LPCVD silicon nitride.

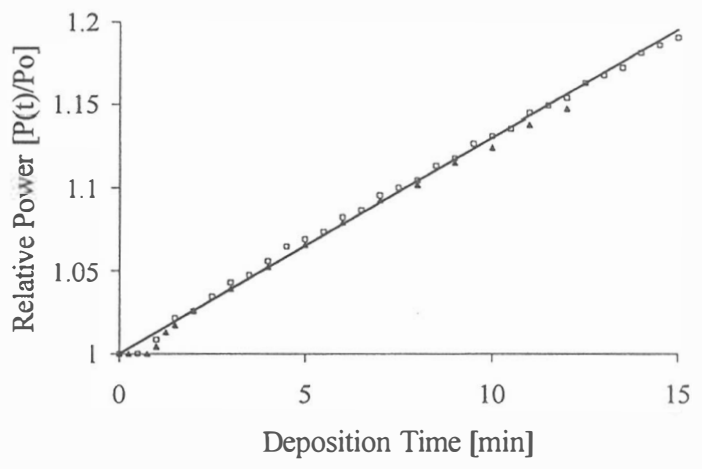

Figure 5. Relative power provided to a microbridge by the control circuit in a CW bias-mode $\mu-C V D$ experiment. $P_{o}$ is the power required prior to metal deposition. Depositions for $12 \mathrm{~min}$ (filled triangles), and $15 \mathrm{~min}$ (open squares) are shown, along with a linear fit to the latter data. For both depositions, the bridge resistance was maintained at $1.4 R_{o}$, where $R_{0}$ is its roomtemperature resistance.

\section{EXPERIMENTAL RESULTS AND DISCUSSION}

The relative power provided by the control circuit to maintain constant $(\mathrm{CW})$ resistance during $\mathrm{Pt}$ deposition is shown in Figure 5. Two depositions of 12 and 15 min total time on two separate devices are shown. These data can be described by Eq. (1). Initially, the power does not increase due to the fact that although Pt is being deposited, the film is not yet continuous and carries no additional heat to the bridge ends. With increasing deposition time, however, the Pt nucleation sites connect and the film begins to conduct heat; this change is evident at $0.75 \mathrm{~min}$ into the deposition. Subsequently, growth is nearly linear in time and the slope is precisely that given by the left-hand side of Eq. (2).

Following deposition, the device with a 12 min deposition was cross-sectioned in four places along its length using a focused ion beam (FIB). A metal thickness distribution was thereby 
obtained, and from this an average metal thickness of $0.67 \mu \mathrm{m}$ was derived. One such FIB cross section is shown in Figure 2.

Deposited Pt significantly increases the heat capacity of the microbridge/metal system, introducing a measurable delay in the transient response of the system to a heating pulse. The two curves shown at the top of Figure 6 are the measured response of a microbridge to a square heating pulse (a) before and (b) after a 12 min $\mathrm{CW}$ deposition. (Note that this deposition was performed at a bridge resistance of 1.4 times the room-temperature value for the bridge, the same conditions under which the $\mathrm{CW}$ depositions of Figure 5 were performed.) The system's response after metal deposition is delayed with respect to that before. Furthermore, in the steady state, the power required after deposition is greater than that before; this is the same effect as that already described for the $\mathrm{CW}$ depositions above.

\section{SIMULATION DETAILS}

The basic electro-thermal model of a microbridge is based on the work of Mastrangelo [7], where the temperature of an element of the bridge is determined by Joule heat gain, and losses due to (a) conduction along the bridge, (b) conduction through the air gap from the hot bridge to the underlying substrate, (c) convection, and (d) radiation. The length of an element is $L / N$ for a bridge of length $L$ divided into $N$ elements.

Using electrical analogues of thermal parameters, components representing the various heat-loss mechanisms and Joule heating gains were implemented in PSPICE ${ }^{\mathrm{TM}}$, as diagrammed in Figure 7. Voltage-controlled current sources (VCCS) were used to make the magnitude of convective and radiative losses and Joule heat gains in a given element dependent on the temperature (voltage) of the node. In this way, complex functional dependencies, such as the fourth-order temperature dependence of radiation, were easily implemented. Secondly, temperature-variable thermal and electrical resistors were constructed from a series combination of a voltage-controlled voltage source and an ammeter (a $0-\mathrm{V}$ independent source in PSPICE $^{\mathrm{TM}}$ ). This is possible since, by Ohm's Law, resistance and voltage are proportional. The output of a VCVS for a given element (the element resistance) is directly proportional to the heating current into that element, as measured by the ammeter. The thermal resistance of an element is also inversely proportional to the thermal conductivity defined in Eq. (4).

Mastrangelo's model assumed that the temperature of the silicon substrate below the device remains constant, regardless of device temperature. In fact, since the substrate is not an ideal heat sink, the temperature in the vicinity of the microbridge increases as the microbridge is heated. This effect is greatest at the microbridge ends, as most of the heat generated within the device is conducted along its length and deposited at its extremities. This leads to localized heating at the ends of a microbridge and increased resistance to heat flow there. By analogy to electrical spreading resistance [8], this effect has been called "thermal spreading resistance", and can be modeled in SPICE by placing additional thermal resistance between the bridge ends and the substrate $[\mathbf{5 , 6 ]}$.

With measured values of the thermal conductivity, $K_{b}$, and heat capacity, $C_{b}$, of polysilicon inserted, the SPICE model just described accurately predicts the temperature profile, currentvoltage characteristics, and transient response of microbridges without deposited metal $[\mathbf{5 , 6 ]}$. To simulate the effects of deposited metal on the steady-state heating characteristics of the bridge/metal system, a thermal conductivity given by Eq. (4) is required. For transient analysis of the system, the elemental heat capacity, $C_{m}$, of the deposited metal, given by Eq. (5), is also required and was added in parallel to $C_{b}$. Finally, to simulate the coupled response of the system and control circuit, a circuit model for the control circuit of Figure 3 was constructed and connected to the electrothermal model of the system.

\section{SIMULATION RESULTS}

Simulation results for the coupled model consisting of the control circuit and bridge/metal system are described in this section. The growth rate, as defined by Eq. (3), was first predicted. The first factor there is given experimentally by the slope of the line in Figure 5. The second factor, the rate of change of $P_{m}$ with $z_{m}$, is easily determined with the SPICE model, since the composite thermal conductivity described by Eq. (4) introduces the effects of $z_{m}$ and $f$ on the steady-state behavior of the system. With this in place, one simply selects values of $f, R$, and $z_{m}$ and the model calculates the corresponding power. From the $15 \mathrm{~min}$ deposition at $R=1.4 R_{o}$ in Figure $5, d P_{m} / d t=0.82 \mathrm{~mW} / \mathrm{min}$. For this same set point and $f=1 / 3$, SPICE calculations yield $d P_{m} / d z_{m}=14 \mathrm{~mW} / \mu \mathrm{m}$. From Eq. (3), then, a Pt growth rate of $59 \mathrm{~nm} / \mathrm{min}$ is obtained. By way of comparison, this rate implies a thickness of $0.7 \mu \mathrm{m}$ for a 12 min deposition, in agreement with the measured value of $0.67 \mu \mathrm{m}$ obtained by FIB cross sections.

The transient response of the system to a heating pulse was next simulated, and a comparison with experiment is given in Figure 6 . The two curves in each graph of Figure 6 represent the transient response (a) before, and (b) after a 12 min CW deposition at $1.4 R_{o}$. Note that this was the same deposition used to evaluate the $\mathrm{CW}$ results above. One would expect, then, that the value of $f$ obtained here should be close to $1 / 3$. In fact, in the simulation of Figure 6 values of $f=1 / 3$ and $z_{m}=0.7 \mu \mathrm{m}$ were used for comparison with the $\mathrm{CW}$ results. The simulated heating delay (measured at $40 \mathrm{~mW}$ ) was $0.29 \mathrm{~ms}$, a value $15 \%$ smaller than the measured value of $0.35 \mathrm{~ms}$. Using $f=0.37$, however, yields the correct heating delay of $0.35 \mathrm{~ms}$. This value of $f$ is within $10 \%$ of that used in the continuous set-point simulation. Finally, it should be noted that the simulated and measured steady-state heating difference before and after deposition (shown as $P_{c}$ in Figure 6), agree within $10 \%$.

\section{SUMMARY AND CONCLUSIONS}

The relatively large increase in the heat conductance and heat capacity of a microbridge due to $\mu-C V D$ metal can be detected in real time as the metal is being deposited through a variation in the heating power provided to the microbridge/metal system. A novel constant-resistance control circuit was presented that can provide either a continuous or pulsed heating bias to the microbridge during deposition. During steady-state operation, the change in heat conductance with the deposited metal is obtained, and can be used to determine the film thickness in situ. On the other hand, during pulsed biasing, the increase in heat capacity with film deposition is extracted and can also be used for in-situ determination of the film thickness.

The techniques described here are applicable to in-situ monitoring of $\mu$-CVD on microhotplates as well, and do not require any additional microfabrication to produce four-point resistance-based thickness probes on the device. Finally, in-situ detection of the $\mu$-CVD of dielectrics on microheater platforms should also be possible using pulsed biasing, since this technique only requires changes in the heat capacity of the system for detection.

The principal advantage of SPICE modeling over other numerical methods is the ability to simultaneously simulate the electro-thermal behavior of a device and its control/sense electronics. Such a coupled model was presented for a microbridge with deposited metal and its constant resistance control circuit. Simulations predicted within $10 \%$ the measured values of the thickness of $\mu$-CVD metal in both pulsed- and CWdeposition bias modes. 

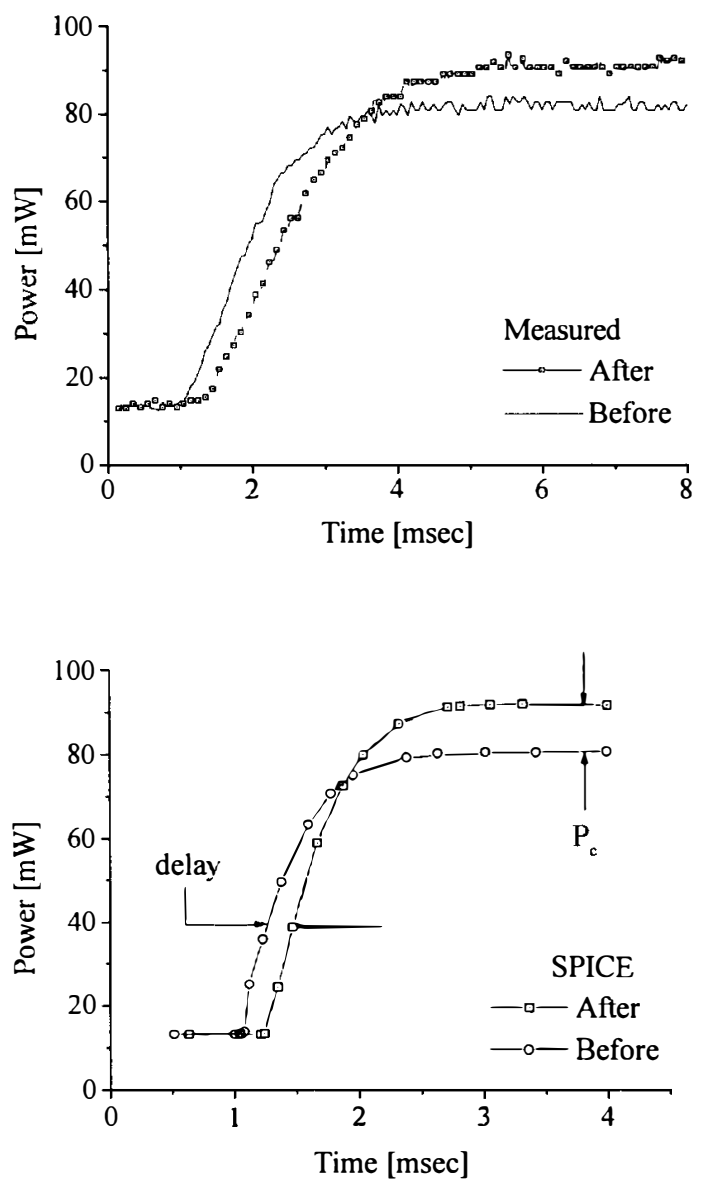

Figure 6. The measured (top) and simulated (bottom) response of a 100- $\mu \mathrm{m}$ bridge to a $1.3 R_{o}$ square heating pulse, where $R_{o}$ is the room-temperature resistance of the bridge. The power indicated is that required to maintain $1.3 R_{\sigma}$. Time response both "before" and "after" a $0.7 \mu \mathrm{m}$ thick Pt deposition are shown. Note that heating was initiated at the same time both before and after deposition. Simulation results are for $f=0.33$.

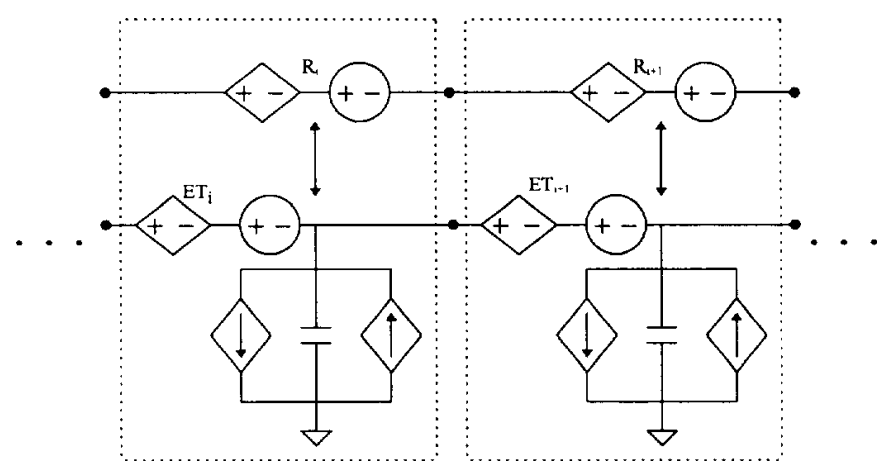

Figure 7. Elements ' $i$ ' and ' $i+1$ ' of the bridge in SPICE. The electrical resistors, ' $R$ ', are coupled to the thermal model at the points indicated by double arrows. The series combination of $a$ $V C V S$ and $0-V$ independent source functions as a temperaturedependent resistor. Joule heating (upward-pointing arrow) and losses (downward-pointing arrow) are modeled with VCCSs. The heat capacity of an element is represented by a capacitor.

\section{ACKNOWLEDGMENTS}

Sandia is a multiprogram laboratory operated by Sandia Corporation, a Lockheed Martin Company, for the United States Department of Energy under contract DE-AC04-94AL85000. The microbridges shown herein were produced at Sandia's Microelectronics Development Laboratory. The excellent technical assistance of the engineers and operators of the MDL is gratefully acknowledged. (Information on the MDL can be found at http://www.mdl.sandia.gov/Micromachine.) Finally, special thanks goes to Alan W. Staton for assistance with catalyst deposition.

\section{REFERENCES}

1. Semancik, R. E. Cavicchi, K. G. Kreider, J. S. Suehle, P. Chaparala, "Selected-Area Deposition of Multiple Active Films for Conductometric Microsensor Arrays", 8th International Conference on Solid-State Sensors and Actuators and Eurosensors IX, Digest of Technical Papers, 1, 831 (1995).

2. S. Majoo, J. W. Schwank, J. L. Gland, and K. D. Wise, "A Selected-Area CVD Method for Deposition of Sensing Films on Monolithically Integrated Gas Detectors", IEEE Electron Device Letters, 16, 217 (1995).

3. R. P. Manginell, J. H. Smith, A. J. Ricco, D. J.Moreno, R. C. Hughes, R. J. Huber, S. D. Senturia, "Selective, Pulsed CVD of Platinum on Microfilament Gas Sensors", Tech. Digest 1996 Sol.State Sensor and Actuator Workshop, Transducers Research Foundation, Cleveland (1996), pp. 53 - 56.

4. S. Majoo, J. L. Gland, K. D. Wise, and J. W. Schwank, "A Silicon Micromachined Conductometric Gas Sensor with a Maskless Pt Sensing Film Deposited by Selected-Area CVD", Sensors and Actuators B, 35 - 36, 312 (1996).

5. R. P. Manginell, J. H. Smith, and A. J. Ricco, "An Overview of Micromachined Platforms for Thermal Sensing and Gas Detection", SPIE's $4^{\text {th }}$ Annual Symposium on Smart Structures and Materials, San Diego, 3/3-6/97.

6. R. P. Manginell, "Polycrystalline-Silicon Microbridge Combustible Gas Sensor", Ph.D. Dissertation, University of New Mexico, 1997.

7. C. H. Mastrangelo, "Thermal Applications of Microbridges", Ph.D. Dissertation, U. C. Berkeley, 1991.

8. S. M. Sze, "Physics of Semiconductor Devices" $2^{\text {nd }}$ Ed., John Wiley \& Sons, New York (1981), p. 217. 